\title{
Translation Invariant States in Quantum Mechanics
}

\author{
R. Beaume \\ Université d'Aix-Marseille II, U.E.R. de Marseille-Luminy, France \\ J. Manuceau and A. Pellet \\ Université de Provence, Marseille, France \\ M. Sirugue \\ Chargé de Recherches C.N.R.S., CPT Marseille, France
}

Received December 3, 1973

\begin{abstract}
We give a complete description of the states of the C.C.R. algebra for a finite number of degrees of freedom which are invariant with respect to subgroups of the translation group of phase space. We make precise some well-known results of quantum mechanics such as Bloch theorem.
\end{abstract}

\section{§ I. Introduction}

In this paper we shall deal with the algebra $\overline{\Delta(\boldsymbol{G}, \xi)}$ of canonical commutation relations (C.C.R.) introduced in [2] (see also [3]) where $\boldsymbol{G}$ is an abelian group and $\xi$ a bicharacter [Definition (2.1)]; for $\boldsymbol{G}=\mathbb{R}^{2 N}$ and $\xi\left((x, p),\left(x^{\prime}, p^{\prime}\right)\right)=\exp \left(-\frac{i}{2} \sum_{i=1}^{n} x_{i} p_{i}^{\prime}-x_{i}^{\prime} p_{i}\right),(x, p) \in \mathbb{R}^{2 N}, \overline{\Delta\left(\mathbb{R}^{2 N}, \xi\right)}$ is the uniform closure of the $*$-algebra of the finite linear combinations of Weyl operators $\delta_{(x, p)}$. The interest of this algebra is that it has a large number of states; namely, any normalized linear positive function on the linear combinations of Weyl operators extends to a (unique) state of $\overline{\Delta(\boldsymbol{G}, \xi)}$.

Translations by $\boldsymbol{g}$ in the phase space $\boldsymbol{G}$ are represented by a *automorphism $\tau_{\boldsymbol{g}}$ of $\overline{\Delta(\boldsymbol{G}, \bar{\xi})}$ which is inner:

$$
\tau_{\boldsymbol{g}} \delta_{\boldsymbol{g}^{\prime}}=\delta_{\boldsymbol{g}} \delta_{\boldsymbol{g}^{\prime}} \delta_{\boldsymbol{g}}^{-1}=\xi\left(\boldsymbol{g}, \boldsymbol{g}^{\prime}\right)^{2} \delta_{\boldsymbol{g}^{\prime}}, \quad \boldsymbol{g}, \boldsymbol{g}^{\prime} \in \boldsymbol{G} .
$$

Let $\boldsymbol{H}$ be a subgroup of $\boldsymbol{G}$ and let $\boldsymbol{H}^{\prime}$ be the set of elements $\boldsymbol{g}$ of $\boldsymbol{G}$ such that $\xi(\boldsymbol{g}, \boldsymbol{h})^{2}=1$ for any $\boldsymbol{h} \in \boldsymbol{H}$. Then the invariant states $\omega$ of $\overline{\Delta(\boldsymbol{G}, \xi)}$ with respect to the $\tau_{\boldsymbol{h}}(\boldsymbol{h} \in \boldsymbol{H})$ are those states for which $\omega\left(\delta_{\boldsymbol{g}}\right)=0$ if $\boldsymbol{g} \notin \boldsymbol{H}^{\prime}$ [Proposition (2.13)]; conversely, let $\omega$ be a state of $\overline{\Delta\left(\boldsymbol{H}^{\prime}, \xi^{\prime}\right)}$ where $\xi^{\prime}$ is the restriction of $\xi$ to $\boldsymbol{H}^{\prime} \times \boldsymbol{H}^{\prime}$; then there exists an invariant extension $\bar{\omega}$ of $\omega$ to $\overline{\Delta(\boldsymbol{G}, \xi)}$ which is given by $\bar{\omega}\left(\delta_{\boldsymbol{g}}\right)=0$ if $\boldsymbol{g} \notin \boldsymbol{H}^{\prime}$ [Proposition (2.14)]. Moreover if $\overline{\Delta\left(\boldsymbol{H}^{\prime}, \xi^{\prime}\right)}$ is abelian and if $\omega$ is pure, $\bar{\omega}$ is the unique (hence pure) extension of $\omega$ to $\overline{\Delta(\boldsymbol{G}, \xi)}$ if and only if $\overline{\Delta\left(\boldsymbol{H}^{\prime}, \xi^{\prime}\right)}$ is maximal abelian [Proposition (2.18)]. 
In Section 3, we specialize $\overline{\Delta\left(\mathbb{R}^{2}, \xi\right)}$, and show that we only need consider closed subgroups of phase space as invariance subgroups [Lemma (2.11)]. Hence except for the trivial cases which are easy to handle, and up to isomorphisms there are three possible cases for $\xi$ abelian subgroups (i.e. for which $\left.\boldsymbol{H} \subset \boldsymbol{H}^{\prime}\right)$;

i) The subgroup of $\mathbb{R}^{2}$ is $\mathbb{R} \times\{0\}$; invariant pure states $\omega$ of $\overline{\Delta\left(\mathbb{R}^{2}, \xi\right)}$ for which $x \rightarrow \omega\left(\delta_{x, p}\right)$ is continuous are of the form

$$
\begin{aligned}
& \omega_{p_{0}}\left(\delta_{x, p}\right)=0 \quad \text { if } \quad p \neq 0 \\
& \omega_{p_{0}}\left(\delta_{x, p}\right)=\exp \left(i p_{0} x\right) \quad p_{0} \in \mathbb{R} .
\end{aligned}
$$

These states are vector states of a representation of $\overline{\Delta\left(\mathbb{R}^{2}, \xi\right)}$ in a non separable Hilbert space [Theorem (3.6)]. These are the analogues of plane waves of the usual formalism.

ii) The subgroup of $\mathbb{R}^{2}$ is $\mathbb{Z} a \times\{0\}$ where $\mathbb{Z}$ is the group of integers, $a \in \mathbb{R}$. Invariant pure states of $\overline{\Delta\left(\mathbb{R}^{2}, \xi\right)}$ for which $x \rightarrow \omega\left(\delta_{x, p}\right)$ is continuous are of the form

$$
\begin{aligned}
\omega\left(\delta_{x, p}\right) & =0 \text { if } \quad p \neq \frac{2 \pi m}{a} \\
\omega\left(\delta_{x, \frac{2 \pi m}{a}}\right) & =e^{i q x} e^{\frac{i \pi m x}{a}} \sum_{n \in \mathbb{Z}} \overline{f(n)} e^{\frac{2 i \pi n x}{a}} f(n+m) \\
q & \in\left[0, \frac{2 \pi}{a}\right] \quad f \in l_{2}(\mathbb{Z})
\end{aligned}
$$

[Bloch theorem (3.13)]. These states are vector states of the previous representation.

iii) The subgroup of $\mathbb{R}^{2}$ is $\mathbb{Z} a \times \mathbb{Z} \frac{2 \pi}{a}, a \in \mathbb{R}$. Invariant pure states $\omega$ of $\overline{\Delta\left(\mathbb{R}^{2}, \xi\right)}$ are of the form

$$
\begin{aligned}
\omega_{\beta \gamma}\left(\delta_{x, p}\right)= & 0 \quad \text { if } \quad(x, p) \neq\left(n \lambda, \frac{2 \pi m}{\lambda}\right) \\
\omega_{\beta \gamma}\left(\delta_{n \lambda, \frac{2 \pi m}{\lambda}}\right)= & \exp \left(i \pi m n+i \beta n \lambda+i \gamma \frac{2 \pi m}{\lambda}\right) \\
& n, m \in \mathbb{Z}, \quad \beta \in[0,2 \pi[, \quad \gamma \in[0, \lambda[.
\end{aligned}
$$

They are vector states of a unique representation within a non-separable Hilbert space; this representation is disjoint from the previous one [Proposition (3.23)].

Finally we study the action of the group of linear non singular transformations of phase space on the above states and give the conditions of implementation of these transformations [Proposition (3.27)]. 


\section{$\S$ II. Definitions and Elementary Results}

Most of the results of this section are slight generalizations of the corresponding results of Ref. [3]; hence they are in general given without proofs (see also [4]).

The bicharacters we shall define now are of central importance in what follows:

Definition (2.1). Let $\boldsymbol{G}$ be an abelian group: a bicharacter $\xi$ is a function $\xi: \boldsymbol{G} \times \boldsymbol{G} \rightarrow \boldsymbol{T}$ (the group of complex numbers of modulus one) such that:

$$
\begin{aligned}
\xi\left(\boldsymbol{g}, \boldsymbol{g}^{\prime}\right) & =\overline{\xi\left(\boldsymbol{g}^{\prime}, \boldsymbol{g}\right)} \quad \forall \boldsymbol{g}, \boldsymbol{g}^{\prime} \in \boldsymbol{G} \\
\xi\left(\boldsymbol{g}, \boldsymbol{g}_{1}+\boldsymbol{g}_{2}\right) & =\xi\left(\boldsymbol{g}, \boldsymbol{g}_{1}\right) \xi\left(\boldsymbol{g}, \boldsymbol{g}_{2}\right) \quad \forall \boldsymbol{g}, \boldsymbol{g}_{1}, \boldsymbol{g}_{2} \in \boldsymbol{G} .
\end{aligned}
$$

A similar procedure to that in [3] can be used to construct a $*$-algebra $\Delta(\boldsymbol{G}, \xi)$ for $\xi$ a bicharacter.

Definition (2.4). $\Delta(\boldsymbol{G}, \xi)$ where $\boldsymbol{G}$ is an abelian group and $\xi$ is a bicharacter is the set of finite linear combinations ${ }^{1}$ :

$$
a=\sum_{i=1}^{N} \lambda_{i} \delta_{g_{i}} \quad \lambda_{i} \in \mathbb{C}, \boldsymbol{g}_{i} \in \boldsymbol{G}
$$

endowed with the following product and $*$-operation:

$$
\begin{gathered}
\delta_{\boldsymbol{g}} \delta_{\boldsymbol{h}}=\xi(\boldsymbol{g}, \boldsymbol{h}) \delta_{\boldsymbol{g}+\boldsymbol{h}} \\
\delta_{\boldsymbol{g}}^{*}=\xi(\boldsymbol{g}, \boldsymbol{g}) \delta_{-\boldsymbol{g}} .
\end{gathered}
$$

As shown in [3] there exists on $\Delta(\boldsymbol{G}, \xi)$ a completely regular norm (i.e. one satisfying $\left\|a^{*} \boldsymbol{a}\right\|=\|\boldsymbol{a}\|^{2}$ )

$$
\|\boldsymbol{a}\|=\sup _{\omega \in \xi_{\boldsymbol{G}}} \sqrt{\omega\left(\boldsymbol{a}^{*} \boldsymbol{a}\right)} \quad \boldsymbol{a} \in \Delta(\boldsymbol{G}, \boldsymbol{\xi})
$$

where $\xi_{\boldsymbol{G}}$ is the set of positive normalized forms on $\Delta(\boldsymbol{G}, \xi)$.

Moreover it is the unique completely regular norm on $\Delta(\boldsymbol{G}, \xi)$ when $\xi$ is non-degenerate (see below for definition).

We denote by $\overline{\Delta(\boldsymbol{G}, \xi)}$ the completion of $\Delta(\boldsymbol{G}, \xi)$ with respect to this norm.

Any positive linear form (resp. *-representation) of $\Delta(\boldsymbol{G}, \xi)$ extends to a positive linear form (resp. *-representation) of $\overline{\Delta(\boldsymbol{G}, \xi)}([3], 3.4)$.

Let us mention the following lemma:

Lemma (2.8). Let $\boldsymbol{G}$ be an abelian group, $\boldsymbol{H}$ a subgroup, $\xi$ a bicharacter on $\boldsymbol{G}$; denote also by $\xi$ the restriction of $\xi$ to $\boldsymbol{H} \times \boldsymbol{H}$; then

$$
\overline{\Delta(\boldsymbol{H}, \xi)} \subseteq \overline{\Delta(\boldsymbol{G}, \xi)} .
$$

Moreover if $\boldsymbol{H} \varsubsetneqq \boldsymbol{G}$, then $\overline{\Delta(\boldsymbol{H}, \xi)} \subsetneq \overline{\Delta(\boldsymbol{G}, \xi)}$.

\footnotetext{
${ }^{1}$ Notice that the unitary elements $\delta_{\boldsymbol{g}}$ are linearly independent (see [2], 2.2.2).
} 
We shall need in the sequel of the notion of commutant of a subgroup of $\boldsymbol{G}$. So we define

Definition (2.9). Let $\boldsymbol{H}$ be a subgroup of $\boldsymbol{G}$. We define $\boldsymbol{H}^{\prime}$, the commutant of $\boldsymbol{H}$, as

$$
\boldsymbol{H}^{\prime}=\left\{\boldsymbol{g} \in \boldsymbol{G} ; \xi(\boldsymbol{g}, \boldsymbol{h})^{2}=1 \forall \boldsymbol{h} \in \boldsymbol{H}\right\} .
$$

$\boldsymbol{H}$ will be $\xi$-abelian if and only if $\boldsymbol{H} \cong \boldsymbol{H}^{\prime}$ and maximal $\xi$-abelian if and only if $\boldsymbol{H}=\boldsymbol{H}^{\prime}$.

We shall say that $\xi$ is non-degenerate if and only if $G^{\prime}=\{0\}$.

One can easily verify the following properties of the commutant operation:

Lemma (2.10). Let $\boldsymbol{G}$ be an abelian group; $\xi$ a bicharacter, $\boldsymbol{H}, \boldsymbol{H}_{1}$ and $\boldsymbol{H}_{2}$ subgroups of $\boldsymbol{G}$. Then:
i. $\boldsymbol{H}^{\prime}$ is a subgroup.
ii. $\boldsymbol{H} \subseteq \boldsymbol{H}^{\prime \prime}$.
iii. $\boldsymbol{H}^{\prime}=\boldsymbol{H}^{\prime \prime \prime}$.
iv. $\boldsymbol{H}_{1} \subseteq \boldsymbol{H}_{2} \Rightarrow \boldsymbol{H}_{2}^{\prime} \cong \boldsymbol{H}_{1}^{\prime}$.

The next lemma will be of some importance in the last section.

Lemma (2.11). Let $\boldsymbol{G}$ be an abelian topological group; let $\xi$ be a bicharacter such that the function $\boldsymbol{g} \rightarrow \xi\left(\boldsymbol{g}, \boldsymbol{g}^{\prime}\right)$ is continuous $\forall \boldsymbol{g}^{\prime} \in \boldsymbol{G}$.

Then if $\boldsymbol{H}$ is a subgroup of $\boldsymbol{G}, \boldsymbol{H}^{\prime}=(\overline{\boldsymbol{H}})^{\prime}$ where $\overline{\boldsymbol{H}}$ denotes the closure of $\boldsymbol{H}$.

Finally we denoted by $\tau$ the canonical injection of the group $\boldsymbol{G}$ into the inner $*$-automorphisms of $\overline{\Delta(\boldsymbol{G}, \xi)}$

$$
\tau_{g}\left(\delta_{\boldsymbol{h}}\right)=\xi(\boldsymbol{g}, \boldsymbol{h})^{2} \delta_{\boldsymbol{h}}
$$

An easy generalization of the previous results can be made for a general group (non-abelian), but in view of the applications of the last section and of the simplification of the notations we just mention it.

Now we shall give some easy results about extensions of states on subalgebras. Proofs are standard or even simplified by the topology (viz. the discrete topology chosen on $\boldsymbol{G}$ ) so in general they are not given.

Lemma (2.13). Let $\boldsymbol{G}$ be an abelian group, $\xi$ a bicharacter, $\boldsymbol{H}$ a subgroup of $\boldsymbol{G}, \tau$ the canonical injection of $\boldsymbol{H}$ into the *-automorphisms group of $\overline{\Delta(\boldsymbol{G}, \xi)}$; then if $\omega$ is a state of $\overline{\Delta(\boldsymbol{G}, \xi)}$,

$$
\omega \circ \tau_{\boldsymbol{h}}=\omega \quad \forall \boldsymbol{h} \in \boldsymbol{H} \Rightarrow \omega\left(\delta_{\boldsymbol{g}}\right)=0 \quad \forall \boldsymbol{g} \notin \boldsymbol{H}^{\prime} .
$$

The next result ensures the existence of an invariant state. 
Theorem (2.14). Let $\boldsymbol{G}$ be an abelian group, $\xi$ a bicharacter, $\boldsymbol{H}$ a subgroup of $\boldsymbol{G}$; let $\omega$ be a positive linear form on $\Delta(\boldsymbol{H}, \xi)$; then $\bar{\omega}$ defined as

$$
\begin{array}{ll}
\bar{\omega}\left(\delta_{\boldsymbol{h}}\right)=\omega\left(\delta_{\boldsymbol{h}}\right) & \forall \boldsymbol{h} \in \boldsymbol{H} \\
\bar{\omega}\left(\delta_{\boldsymbol{g}}\right)=0 & \forall \boldsymbol{g} \notin \boldsymbol{H}
\end{array}
$$

gives a positive linear form on $\Delta(G, \xi)$.

Using the remark before Lemma (2.8) one has the following corollary.

Corollary (2.15). Any state $\omega$ of the subalgebra $\overline{\Delta(\boldsymbol{H}, \xi)}$ of $\overline{\Delta(\boldsymbol{G}, \xi)}$ can be extended to a state $\bar{\omega}$ of $\overline{\Delta(\boldsymbol{G}, \xi)}$ in the following way

$$
\begin{array}{llll}
\bar{\omega}(\boldsymbol{a})=\omega(\boldsymbol{a}) & \text { if } & \boldsymbol{a} \in \overline{\Delta(\boldsymbol{H}, \xi)} \\
\bar{\omega}\left(\delta_{\boldsymbol{g}}\right)=0 & \text { if } & \boldsymbol{g} \notin \boldsymbol{H} .
\end{array}
$$

The next theorem gives an explicit realization of the representation associated to the extension $\bar{\omega}$.

Theorem (2.16). Let $\boldsymbol{G}$ be an abelian group, $\xi$ a bicharacter and $\boldsymbol{H}$ a subgroup of $\boldsymbol{G}$. Let $\omega$ be a state of $\overline{\Delta(\boldsymbol{H}, \xi)},\left(\mathscr{H}_{\omega}, \Omega_{\omega}, \pi_{\omega}\right)$ the corresponding Gelfand-Naimark-Segal (G.N.S.) triplet. Let $\bar{\omega}$ be the extension of $\omega$ to $\overline{\Delta(\boldsymbol{G}, \xi)}$ previously described. Let $S$ be an application of $\boldsymbol{G}$ into $\boldsymbol{G}$ such that

$$
\begin{aligned}
S(0) & =0 \\
S(\boldsymbol{g}+\boldsymbol{h}) & =S(\boldsymbol{g}) \quad \forall \boldsymbol{g} \in \boldsymbol{G}, \quad \forall \boldsymbol{h} \in \boldsymbol{H} \\
\boldsymbol{g}-S(\boldsymbol{g}) & =T(\boldsymbol{g}) \in \boldsymbol{H} \quad \forall \boldsymbol{g} \in \boldsymbol{G} .
\end{aligned}
$$

Let $\mathscr{H}_{\bar{\omega}}$ be the Hilbert space $l_{2}(\boldsymbol{G} / \boldsymbol{H}) \otimes \mathscr{H}_{\omega},[\boldsymbol{g}]$ the function of $l_{2}(\boldsymbol{G} / \boldsymbol{H})$ which is one on the class of $\mathbf{g}$ and zero otherwise.

Then $\bar{\pi}$ defined as the linear transformation such that

$$
\bar{\pi}\left(\delta_{\boldsymbol{k}}\right)\left[S(\boldsymbol{g}) \otimes \pi_{\omega}\left(\delta_{T(\boldsymbol{g})}\right) \Omega_{\omega}=\Xi(\boldsymbol{k}, \boldsymbol{g})[S(\boldsymbol{g}+\boldsymbol{k})] \otimes \pi_{\omega}\left(\delta_{T(\boldsymbol{g}+\boldsymbol{k})}\right) \Omega_{\omega}\right.
$$

where

$$
\Xi(\boldsymbol{k}, \boldsymbol{g})=\xi(\boldsymbol{k}, \boldsymbol{g}) \xi(S(\boldsymbol{g}), T(\boldsymbol{g})) \overline{\xi(S(\boldsymbol{g}+\boldsymbol{k}), T(\boldsymbol{g}+\boldsymbol{k}))}
$$

extends to a *-representation of $\overline{\Delta(\boldsymbol{G}, \xi)}$ in $l^{2}(\boldsymbol{G} / \boldsymbol{H}) \otimes \mathscr{H}_{\omega}$ with cyclic vector $[0] \otimes \Omega_{\omega}$; and it is unitarily equivalent to the representation constructed from $\bar{\omega}$ by the usual G.N.S. procedure.

First notice that $[S(\boldsymbol{g})] \otimes \pi_{\omega}\left(\delta_{\boldsymbol{h}}\right) \Omega_{\omega}=\left[S\left(\boldsymbol{g}^{\prime}\right)\right] \otimes \pi_{\omega}\left(\delta_{T\left(\boldsymbol{g}^{\prime}\right)}\right) \Omega_{\omega}$ where $\boldsymbol{g}^{\prime}=S(\boldsymbol{g})+\boldsymbol{h}, \boldsymbol{g} \in \boldsymbol{G}, \boldsymbol{h} \in \boldsymbol{H}$.

Hence $\bar{\pi}\left(\delta_{k}\right)$ is defined over a dense set of vectors; moreover $\bar{\pi}$ is well-defined since if

$$
\Phi=[S(\boldsymbol{g})] \otimes\left(\sum_{i=1}^{n} \lambda_{i} \pi_{\omega}\left(\delta_{\boldsymbol{h}_{i}}\right)\right) \Omega_{\omega}=0
$$


then

$$
\begin{aligned}
\bar{\pi}\left(\delta_{\boldsymbol{k}}\right) \Phi & =\Xi(\boldsymbol{k}, S(\boldsymbol{g}))[S(\boldsymbol{g}+\boldsymbol{k})] \otimes \pi_{\omega}\left(\delta_{T(S(g)+\boldsymbol{k})}\right)\left(\sum_{i=1}^{n} \lambda_{i} \pi_{\omega}\left(\delta_{h_{i}}\right)\right) \Omega_{\omega} \\
& =0 ;
\end{aligned}
$$

the representation character of $\bar{\pi}$ follows from an easy but tedious calculation.

Corollary (2.17). $\bar{\pi}$ is reducible if $\pi_{\omega}$ is reducible.

The restriction of $\bar{\pi}$ to $\overline{\Delta(\boldsymbol{H}, \xi)}$ is given as one can easily compute by

$$
\bar{\pi}\left(\delta_{\boldsymbol{h}}\right)[S(\boldsymbol{g})] \otimes \pi_{\omega}\left(\delta_{T(\boldsymbol{g})}\right) \Omega_{\omega}=\xi(\boldsymbol{h}, S(\boldsymbol{g}))^{2}[S(\boldsymbol{g})] \otimes \pi_{\omega}\left(\delta_{\boldsymbol{h}}\right) \pi_{\omega}\left(\delta_{T(\boldsymbol{g})}\right) \Omega_{\omega}
$$

for $\boldsymbol{h} \in \boldsymbol{H}$.

The next results are connected with the uniqueness and irreducibility of $\bar{\omega}$.

Theorem (2.18). Let $\boldsymbol{G}$ be an abelian group and $\xi$ a bicharacter. Let $\boldsymbol{H}$ be a subgroup of $\boldsymbol{G}$ such that $\boldsymbol{H} \subset \boldsymbol{H}^{\prime}$; then if $\omega$ is a pure state of $\overline{\Delta(\boldsymbol{H}, \xi)}$, $\bar{\omega}$ is the unique extension of $\omega$ to $\overline{\Delta(\boldsymbol{G}, \xi)}$ if and only if $\boldsymbol{H}=\boldsymbol{H}^{\prime}$, and consequently is pure.

Proof. Since $\overline{\Delta(\boldsymbol{H}, \xi)}$ is abelian, $\omega$ is a character, hence

$$
\left|\omega\left(\delta_{\boldsymbol{h}}\right)\right|=1 .
$$

Moreover let $\bar{\omega}$ be an extension of $\omega$ to $\overline{\Delta(\boldsymbol{G}, \xi)}$ [see Corollary (2.15)] then:

$$
\bar{\omega}\left(\delta_{\boldsymbol{g}} \delta_{\boldsymbol{h}} \delta_{\boldsymbol{g}}^{*}\right)=\xi(\boldsymbol{g}, \boldsymbol{h})^{2} \omega\left(\delta_{\boldsymbol{h}}\right)
$$

Let $(\bar{\Omega}, \bar{\pi}, \overline{\mathscr{H}})$ be the G.N.S. triplet associated to $\bar{\omega}$; then from the previous relation

hence

$$
\bar{\pi}\left(\delta_{\boldsymbol{h}}\right) \bar{\pi}\left(\delta_{\boldsymbol{g}}^{*}\right) \bar{\Omega}=\xi(\boldsymbol{g}, \boldsymbol{h})^{2} \omega(\boldsymbol{h}) \bar{\pi}\left(\delta_{\boldsymbol{g}}^{*}\right) \bar{\Omega},
$$

$$
\begin{aligned}
\bar{\omega}\left(\delta_{\boldsymbol{g}}\right) & =\left(\bar{\Omega} \mid \bar{\pi}\left(\delta_{\boldsymbol{g}}\right) \bar{\Omega}\right) \\
& =\overline{\omega(\boldsymbol{h})}\left(\bar{\Omega} \mid \bar{\pi}\left(\delta_{\boldsymbol{g}}\right) \bar{\pi}\left(\delta_{\boldsymbol{h}}\right) \bar{\Omega}\right) \\
& =\xi(\boldsymbol{g}, \boldsymbol{h})^{2}\left(\bar{\Omega} \mid \bar{\pi}\left(\delta_{\boldsymbol{g}}\right) \bar{\Omega}\right) ;
\end{aligned}
$$

hence the result. Notice that from $([6], 2.10 .2)$ there exists at least one pure extension of a pure state.

From the previous construction we have the following result:

Corollary (2.19). The representation space corresponding to $\bar{\omega}$ is separable if and only if $\mathscr{H}_{\omega}$ is separable and $\boldsymbol{G} / \boldsymbol{H}$ is at most countable. 


\section{§ III. Applications: Invariant States of the C.C.R. of a Finite Number of Degrees of Freedom with Respect to a $\xi$-Abelian Subgroup of the Phase Space}

In the following we shall be interested in $G=\mathbb{R}^{2 N}$ considered as $\mathbb{R}^{N} \times \mathbb{R}^{N}$; a typical element of $\mathbb{R}^{2 N}$ will be written as

$$
X=\left(x_{1}, \ldots, x_{N}, p_{1}, \ldots, p_{N}\right) \quad x_{i}, p_{i} \in \mathbb{R} .
$$

Moreover we shall deal with the following bicharacter on $\mathbb{R}^{2 N}$

$$
\begin{aligned}
\xi\left(\left(x_{i}, p_{i}\right),\left(x_{i}^{\prime}, p_{i}^{\prime}\right)\right) & =\exp \left(-\frac{i}{2} \sigma\left(X, X^{\prime}\right)\right) \\
& =\exp \left(-\frac{i}{2}\left(X \mid J X^{\prime}\right)\right)=\exp \left(-\frac{i}{2} \sum_{i=1}^{N} x_{i} p_{i}^{\prime}-x_{i}^{\prime} p_{i}\right)
\end{aligned}
$$

where $\left(X \mid X^{\prime}\right)$ denotes the euclidean scalar product on $\mathbb{R}^{2 N}$ and $\sigma$ and $J$ have obvious definitions:

$$
J\left(x_{i}, p_{i}\right)=\left(p_{i},-x_{i}\right) .
$$

This bicharacter is continuous with respect to the natural topology of $\mathbb{R}^{2 N}$ hence by (2.11) we only shall consider closed subgroups of $\mathbb{R}^{2 N}$.

For the sake of physical applications we have in mind, we shall consider only $\xi$-abelian subgroups of $\mathbb{R}^{2 N}$; moreover we shall treat only the case $N=1$; the general case can be dealt with by the following lemma which uncouples the different degrees of freedom.

Lemma (3.3). Let $\Gamma$ be a lattice in $\mathbb{R}^{2 N}$ and $\Gamma^{\prime}$ its reciprocal lattice, viz. $\Gamma$ is of the form

with

$$
\begin{array}{cc}
a \in \Gamma \Leftrightarrow a=\sum_{i=1}^{N} m_{i} a_{i} & m_{i} \in \mathbb{Z} \\
a^{\prime} \in \Gamma^{\prime} \Leftrightarrow a^{\prime}=\sum_{i=1}^{N} n_{i} \alpha_{i} & n_{i} \in \mathbb{Z}
\end{array}
$$

$$
\begin{array}{ll}
\sigma\left(a_{i}, a_{j}\right)=\sigma\left(\alpha_{i}, \alpha_{j}\right)=0 & \forall i, j=1 \ldots N \\
\sigma\left(a_{i}, \alpha_{j}\right)=2 \pi \delta_{i j} & \forall i, j=1 \ldots N
\end{array}
$$

$\left(\Gamma \cup \Gamma^{\prime}\right.$ defines the most general $\xi$-abelian discrete subgroup of $\left.\mathbb{R}^{2 N}\right)$. Then there exists a linear non-singular transformation $M$ such that

$$
\begin{aligned}
& \sigma(M X, M Y)=\sigma(X, Y) \quad X, Y \in \mathbb{R}^{2 N} \\
& \left(M a_{i}\right)=(0, \ldots 1, \ldots 0, \underbrace{0, \ldots 0}_{i \text {-th place }}) \\
& \left(M \alpha_{i}\right)=(\underbrace{0, \ldots 0}_{N \text { times }}, 0, \ldots 2 \pi, \ldots 0) .
\end{aligned}
$$


The existence of $M$ is ensured by the isomorphism of symplectic spaces of the same finite dimension. For the sake of practical applications it is worth while to give an explicit form of $M$

$$
\begin{aligned}
& M_{i j}=-\left(J a_{i}\right)_{j} \quad \text { if } \quad i>N \\
& M_{i j}=\frac{1}{2 \pi}\left(J \alpha_{i}\right)_{j} \quad \text { if } \quad i \leqq N .
\end{aligned}
$$

Classification of closed subgroups of $\mathbb{R}^{2}$ is well-known; the next table give the ones for which the restriction of $\xi$ is \pm 1 , except for the trivial subgroup.

\begin{tabular}{lllll}
\hline Subgroup & $\xi$-commutant & $\begin{array}{l}\text { Interpretation } \\
\text { of } \boldsymbol{H}\end{array}$ & Property of $\boldsymbol{H}^{\prime}$ & $\begin{array}{l}\text { Corresponding } \\
\text { invariant state }\end{array}$ \\
\hline $\mathbb{R} \times\{0\}$ & $\boldsymbol{H}^{\prime}=\boldsymbol{H}^{\prime \prime}=\boldsymbol{H}$ & Translations & Max-abelian & Plane waves \\
$\mathbb{Z} \times\{0\}$ & $\boldsymbol{H}^{\prime}=\mathbb{R} \times \mathbb{Z}$ & $\begin{array}{l}\text { Discrete } \\
\text { translations }\end{array}$ & $\begin{array}{l}\text { Contains } \mathbb{R} \times\{0\} \\
\text { which is max-abelian }\end{array}$ & Bloch waves \\
$\mathbb{Z} \times \mathbb{Z}$ & $\boldsymbol{H}^{\prime \prime}=\boldsymbol{H}$ & Max-abelian & Zak waves \\
\hline
\end{tabular}

We shall study these three cases separately.

\section{1st Case: Plane Waves}

Proposition (3.5). Let $\left\{\tau_{a} ; a \in \mathbb{R}\right\}$ be the group of $*$-automorphisms of $\overline{\Delta\left(\mathbb{R}^{2}, \xi\right)}$ such that

$$
\tau_{a} \delta_{x, p}=e^{-i a p} \delta_{x, p} \quad \forall a \in \mathbb{R} .
$$

Every pure state $\omega$ of $\overline{\Delta\left(\mathbb{R}^{2}, \xi\right)}$ such that

$$
x \rightarrow \omega\left(\delta_{x, p}\right) \quad \text { is continuous }
$$

and invariant with respect to $\tau_{a}(\forall a \in \mathbb{R})$ is a state $\omega_{p_{0}}$ defined by

$$
\begin{aligned}
& \omega_{p_{0}}\left(\delta_{x, p}\right)=0 \quad \text { if } \quad p \neq 0 \\
& =e^{i p_{0} x} \text { if } p=0 .
\end{aligned}
$$

The proof is obvious and we shall give in the next proposition the structure of the representation.

Proposition (3.6). Let $\omega_{p_{0}}$ be a state defined as

$$
\begin{aligned}
& \omega_{p_{0}}\left(\delta_{x, p}\right)=0 \quad \text { if } \quad p \neq 0 \\
& =e^{i p_{0} x} \text { if } p=0 .
\end{aligned}
$$


Let $\mathscr{H}_{p_{0}}, \pi_{p_{0}}$, and $\Omega_{p_{0}}$ be the canonical Hilbert space, representation and cyclic vector constructed via the G.N.S. theorem. Then

i. $\mathscr{H}_{p_{0}}=l^{2}(\mathbb{R})$ is not separable.

ii. $\pi_{p_{0}}$ is irreducible.

iii. $\forall p^{\prime} \in \mathbb{R} \quad \omega_{p^{\prime}}$ is a vector state of $\pi_{p_{0}}$.

iv. $\pi_{p_{0}}$ is disjoint from any Weyl state.

Proof. i. Is obvious from Theorem (2.16); ii. is clear from (2.18); to prove iii., let us consider $\mid q) \in l_{2}(\mathbb{R})$ such that $\left.\mid q\right)(x)=0$ if $x \neq q$ and |q) $(x)=1$ if $x=q$. Then let $S$ and $T$ be:

$$
\begin{aligned}
& S(x, p)=(0, p) \\
& T(x, p)=(x, 0) .
\end{aligned}
$$

Consequently according to Theorem (2.16)

Hence

$$
\left.\left.\pi_{p_{0}}\left(\delta_{x, p}\right) \mid q\right) \otimes \Omega_{p_{0}}=e^{-\frac{\imath}{2}(p+2 q) x} e^{i p_{0} x} \mid q+p\right) \otimes \Omega_{p_{0}} .
$$

$$
\left.\left.\omega_{p^{\prime}}\left(\delta_{x, p}\right)=\left(\mid p_{0}-p^{\prime}\right) \otimes \Omega_{p_{0}}\left|\pi_{p_{0}}\left(\delta_{x, p}\right)\right| p_{0}-p^{\prime}\right) \otimes \Omega_{p_{0}}\right) .
$$

Moreover $\omega_{p_{0}}$ is disjoint from any Weyl state; indeed if it is not disjoint it is unitarily equivalent to any Weyl pure state, since it is pure, but the usual Schrödinger representation acts within a separable Hilbert space.

Proposition (3.9). Let $\omega_{p_{0}}$ be as previously.

I. $\tau_{a}(\forall a \in \mathbb{R})$ is unitarily implemented within $\pi_{p_{0}}$ by $\pi_{p_{0}}\left(\delta_{a, 0}\right)$ which is strongly continuous in $a$; its spectrum is purely discrete and is the set $\left\{\exp \left(i p^{\prime} a\right) ; p^{\prime} \in \mathbb{R}\right\}$.

II. Let $\left\{\alpha_{t}\right\}_{t \in \mathbb{R}}$ be the group of $*$-automorphisms of $\overline{\Delta\left(\mathbb{R}^{2}, \xi\right)}$ such that

$$
\alpha_{t} \delta_{x, p}=\delta_{x+\frac{t}{m} p, p} m>0, \quad t \in \mathbb{R} ;
$$

$\omega_{p_{0}}$ is $\alpha_{t}$ invariant; $\alpha_{t}$ is unitarily implemented within $\pi_{p_{0}}$ by a strongly continuous group of unitaries whose infinitesimal generator has a discrete spectrum from $-\frac{p_{0}^{2}}{2 m}$ to $\infty$.

Proof. I. Is obvious. In order to prove II we have that $\left(\Omega_{p_{0}} \mid \pi_{p_{0}}\left(\delta_{x_{1}, p_{1}}\right) \pi_{p_{0}}\left(\alpha_{t} \delta_{x_{2}, p_{2}}\right) \Omega_{p_{0}}\right)$

$$
\begin{array}{ll}
=0 & \text { if } \quad p_{1} \neq-p_{2} \\
=\exp \left(i\left(x_{1}+x_{2}\right)\left(p_{0}+\frac{p}{2}\right)-\frac{i t}{m}\left(p_{0} p+\frac{p^{2}}{2}\right)\right) & \text { if } \quad p_{1}=-p_{2}=p
\end{array}
$$

which shows that $\left\{U_{t}, t \in \mathbb{R}\right\}$ the group of unitary operators which implements $\alpha_{t}$ is continuous; moreover that its spectrum extends from $-\frac{p_{0}^{2}}{2 m}$ to $\infty$. 


\section{2nd Case: Bloch Waves}

In this case we shall deal with discrete translations; the invariance group $\Gamma$ is generated by a $*$-automorphism $\tau_{a}$ such that

Hence

$$
\tau_{a} \delta_{x, p}=e^{-i a p} \delta_{x, p} .
$$

$$
\Gamma=\{(n a, 0) ; n \in \mathbb{Z}\} \text {. }
$$

A classical theorem [7], due to Bloch, states that if the hamiltonian of one particle is invariant by discrete translations of the type $n a, n \in \mathbb{Z}$, one can find eigen-solutions of the Schrödinger equation as

$$
\psi(x)=e^{i q x} \mathscr{U}_{q}(x)
$$

where $q \in\left[0, \frac{2 \pi}{a}\left[\right.\right.$ and $\mathscr{U}_{q}(x+a)=\mathscr{U}_{q}(x)$. Let us state the same result in our formalism.

Theorem (Bloch) (3.13). Any pure state $\omega$ of $\overline{\Delta\left(\mathbb{R}^{2}, \xi\right)}$, invariant with respect to $\Gamma$ and such that $x \rightarrow \omega\left(\delta_{x, p}\right)$ is continuous at $x=0$ is of the form

$$
\begin{gathered}
\omega\left(\delta_{x, p}\right)=0 \text { if } \quad p \neq \frac{2 \pi m}{a}, \quad m \in \mathbb{Z} \\
\omega\left(\delta_{\left.x, \frac{2 \pi m}{a}\right)}\right)=e^{i q x} e^{\frac{i \pi m x}{a}} \sum_{n \in \mathbb{Z}} \overline{f(n)} e^{\frac{2 i \pi n x}{a}} f(n+m)
\end{gathered}
$$

where $q \in\left[0, \frac{2 \pi}{a}\left[\right.\right.$ and $f$ is in $l_{2}(\mathbb{Z})$ with $\|f\|_{2}=1$.

We shall prove the necessity of the previous formula through a number of lemmas. The sufficiency will be much easier to prove.

Lemma (3.14). Let $\omega$ be a pure state of $\overline{\Delta\left(\mathbb{R}^{2}, \xi\right)}$ invariant with respect to $\Gamma ; \omega \uparrow$, its restriction to $\overline{\Delta\left(\Gamma^{\prime}, \xi\right)}$, is still a pure state.

$$
\Gamma^{\prime}=\left\{\left(x, \frac{2 \pi m}{a}\right) ; m \in \mathbb{Z}\right\} .
$$

According to Lemma (2.13)

$$
\omega\left(\delta_{x, p}\right)=0 \quad \text { if } \quad(x, p) \notin \Gamma^{\prime} .
$$

Moreover if $\omega \uparrow$ is not pure it has a non-trivial decomposition

$$
\omega \uparrow=\alpha \varrho_{1}+(1-\alpha) \varrho_{2}
$$

and $\omega=\bar{\omega} \uparrow$ has a similar decomposition

$$
\omega=\alpha \bar{\varrho}_{1}+(1-\alpha) \bar{\varrho}_{2}
$$

since the invariant extension of $\omega \uparrow$ is given by Lemma (2.15). 


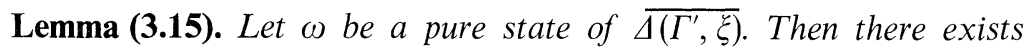
$q \in\left[0, \frac{2 \pi}{a}\left[\right.\right.$ such that $\varrho\left(\tilde{\delta}_{y, \frac{2 \pi m}{a}}\right)=e^{-2 i q y} \omega\left(\delta_{i y, \frac{2 \pi m}{a}}\right)$ for any $y \in[0, a[$ is a pure state of the algebra $\overline{\Delta(\boldsymbol{T} \times \mathbb{Z}, \zeta)}$ with $\varrho\left(\tilde{\delta}_{\frac{a}{2}}, 0\right)=1$. Here $\boldsymbol{T} \times \mathbb{Z}=\left\{\left(y, \frac{2 \pi m}{a}\right) ; y \in[0, a[, m \in \mathbb{Z}\}\right.$ is the abelian group of the torus multiplied by integers (with its natural topology) and

defined by

$$
\zeta:(\boldsymbol{T} \times \mathbb{Z}) \times(\boldsymbol{T} \times \mathbb{Z}) \rightarrow \boldsymbol{T}
$$

$$
\zeta\left(\left(y, \frac{2 \pi m}{a}\right),\left(y^{\prime}, \frac{2 \pi m^{\prime}}{a}\right)\right)=\exp \left(-\frac{2 i \pi}{a}\left(m^{\prime} y-m y^{\prime}\right)\right)
$$

is continuous bicharacter of $\boldsymbol{T} \times \mathbb{Z}$.

Conversely, if $\varrho$ is a (pure) state of the algebra $\overline{\Delta(\boldsymbol{T} \times \mathbb{Z}, \zeta)}$ with $\varrho\left(\tilde{\delta}_{\frac{a}{2}, 0}\right)=1$, then $\omega\left(\delta_{y}, \frac{2 \pi m}{a}\right)=e^{i q y} \varrho\left(\tilde{\delta}_{\frac{y}{2}}, \frac{2 \pi m}{a}\right)$ is a (pure) state of $\bar{\Delta}\left(\Gamma^{\prime}, \xi\right)$.

This lemma is of central importance in the proof of Theorem (3.13). Let us define $q$. Since $\omega$ is pure, it is a character when restricted to the center of $\overline{\Delta\left(\Gamma^{\prime}, \xi\right)}$ which is generated by the $\delta_{n a, 0}, n \in \mathbb{Z}$.

Hence there exists $q \in\left[0, \frac{2 \pi}{a}[\right.$ such that

$$
\omega\left(\delta_{n a, 0}\right)=\exp (-i q n a) \quad n \in \mathbb{Z} .
$$

According to [3], Proposition (3.4) we have only to prove the $\zeta$-positivity of $\varrho$; given $\lambda_{i} \in \mathbb{C}, y_{i} \in\left[0, a\left[, m_{i} \in \mathbb{Z} i=1 \ldots N\right.\right.$

$$
\begin{aligned}
& { }^{1}{ }^{N} u_{i} \lambda_{j} \sum_{i, j=1}^{N} \bar{\lambda}_{i} \lambda_{j} \varrho\left(\tilde{\delta}_{-y_{i}+a \delta_{i}}, \frac{-2 \pi m_{i}}{a} \tilde{\delta}_{y_{j}}, \frac{2 \pi m_{j}}{a}\right) \quad \begin{array}{rlll}
\delta_{i} & =0 & \text { if } & y_{i}=0 \\
& =1 & \text { if } & y_{i}>0
\end{array} \\
& =\sum_{i, j=1}^{N} \bar{\lambda}_{i} \lambda_{j} \exp \left(\frac{2 i \pi}{a}\left(y_{i} m_{j}-y_{j} m_{i}\right)\right) \quad \begin{array}{rlll}
\delta_{i j}=0 & \text { if } & y_{j}-y_{i}+a \delta_{i}<a \\
=1 & \text { if } & y_{j}-y_{i}+a \delta_{i} \geqq a
\end{array} \\
& \cdot \varrho\left(\tilde{\delta}_{-y_{i}+y_{j}+a \delta_{i}-a \delta_{i j}, \frac{2 \pi}{a}\left(m_{J}-m_{\imath}\right)}\right) \\
& =\sum_{i, j=1}^{N} \bar{\lambda}_{i} \lambda_{j} \exp \left(\frac{2 i \pi}{a}\left(y_{i} m_{j}-y_{j} m_{i}\right)-2 i q\left(-y_{i}+y_{j}+a \delta_{i}-a \delta_{i j}\right)\right) \\
& \cdot \omega\left(\delta_{-2\left(y_{t}-y_{j}-a \delta_{t}+a \delta_{i j}\right)}, \frac{2 \pi}{a}\left(m_{j}-m_{\imath}\right)\right) ;
\end{aligned}
$$

using (3.16) the previous expression is reduced to

$$
\begin{aligned}
& \sum_{i, j=1}^{N} \bar{\lambda}_{i} \lambda_{j} e^{-2 i q\left(y_{j}-y_{i}\right)} \exp \left(\frac{2 i \pi}{a}\right.\left.\left(m_{j} y_{i}-m_{i} y_{j}\right)\right) \\
& \cdot \omega\left(\delta_{-2 y_{i}+2 y_{j}, \frac{2 \pi}{a}\left(m_{j}-m_{\imath}\right)}\right) \geqq 0
\end{aligned}
$$

by positivity of $\omega$. 
By a similar computation, it is easy to prove that if $\varrho$ is a state of $\overline{\Delta(\boldsymbol{T} \times \mathbb{Z}, \zeta)}$ with $\varrho\left(\tilde{\delta}_{\frac{a}{2}}, 0\right)=1$, then $\omega$ is a state of $\overline{\Delta\left(\Gamma^{\prime}, \xi\right)}$. Then it follows that $\varrho$ is pure if and only if $\omega$ is pure.

Lemma (3.17). Let $\omega$ be a pure state of $\overline{\Delta\left(\Gamma^{\prime}, \xi\right)}$; then $\varrho$, defined in Lemma (3.15), is continuous with respect to the natural topology of $\boldsymbol{T} \times \mathbb{Z}$ if and only if $x \rightarrow \omega\left(\delta_{x, \frac{2 \pi m}{a}}\right)$ is continuous at $x=0$.

The proof is obvious.

Lemma (3.18). Let $\varrho$ be a pure state of $\overline{\Delta(T \times \mathbb{R}, \zeta)}$ continuous with respect to the natural topology of $\boldsymbol{T} \times \mathbb{Z}$ and such that $\varrho\left(\tilde{\delta}_{\frac{a}{2}}, 0\right)=1$; then there exists $f \in l_{2}(\mathbb{Z}),\|f\|_{2}=1$ such that

$$
\varrho\left(\tilde{\delta}_{y, \frac{2 \pi m}{a}}\right)=e^{\frac{2 i \pi m y}{a}} \sum_{n \in \mathbb{Z}} \overline{f(n)} e^{\frac{4 i \pi n y}{a}} f(n+m) .
$$

This lemma follows from Theorem 1 of $[9]$ (also see $[8,10]$ ). Collecting these results one can see that the formula in Theorem (3.13) is a necessary condition; the sufficiency is almost obvious.

The correspondence of our result to the usual one is obvious; the $f(n)$ in Theorem (3.13) are nothing but the Fourier coefficients of $\mathscr{U}_{q}(x)$ in (3.12).

The states we have defined in Proposition (3.5) are invariant with respect to any translation, hence by $\Gamma$; actually we have the following result:

Proposition (3.19). Let $\omega$ be a pure state of $\overline{\Delta\left(\mathbb{R}^{2}, \xi\right)}$ invariant with respect to $\Gamma$ and such that $x \rightarrow \omega\left(\delta_{x, p}\right)$ is continuous at $x=0$; it is a vector state of $\pi_{p_{0}}$ defined in Proposition (3.6). More precisely

where

$$
\omega\left(\delta_{x, p}\right)=\left(\Phi \mid \pi_{p_{0}}\left(\delta_{x, p}\right) \Phi\right)
$$

$$
\Phi=\sum_{n} f(n) \pi_{0}\left(\delta_{0,-\left(q+\frac{2 \pi n}{a}\right)}\right) \Omega_{0}
$$

The proof requires an obvious calculation.

\section{3rd Case: Zak Waves}

As we saw previously there is a third type of closed $\xi$-abelian subgroups of $\left(\mathbb{R}^{2}, \xi\right)$; namely those isomorphic to $\mathbb{Z}^{2}$; the corresponding lattice is generated by two vectors $a=\left(a_{1}, a_{2}\right)$ and $\alpha=\left(\alpha_{1}, \alpha_{2}\right)$, and the abelianness of the generated group implies that

$$
a_{1} \alpha_{2}-a_{2} \alpha_{1}=2 \pi \text {. }
$$


According to Lemma (3.3) we shall restrict ourselves to the case where

$$
\begin{aligned}
& a=(\lambda, 0) \\
& \alpha=\left(0, \frac{2 \pi}{\lambda}\right) .
\end{aligned}
$$

Definition (3.22). A Zak wave is a state $\omega_{\beta \gamma}$ of C.C.R. such that

$$
\omega_{\beta \gamma}\left(\delta_{x, p}\right)=0 \quad \text { if } \quad(x, p) \neq\left(n \lambda, \frac{2 \pi m}{\lambda}\right)
$$

$\omega_{\beta \gamma}\left(\delta_{n \lambda, \frac{2 \pi m}{\lambda}}\right)=\exp \left(i \beta n \lambda+i \gamma \frac{2 \pi m}{\lambda}\right) \exp (i \pi m n) \quad \beta \in\left[0, \frac{2 \pi}{\lambda}[\gamma \in[0, \lambda[\right.$.

Proposition (3.23). Let $\pi_{\beta \gamma}, \mathscr{H}_{\beta \gamma}, \Omega_{\beta \gamma}$ be the G.N.S. triplet associated to $\omega_{\beta \gamma}$

1) $\omega_{\beta \gamma}$ is pure; $\pi_{\beta \gamma}$ is irreducible.

2) $\mathscr{H}_{\beta \gamma}$ is non-separable.

3) $\forall \beta^{\prime}$ and $\gamma^{\prime}, \omega_{\beta^{\prime} \gamma^{\prime}}$ is a vector state of $\pi_{\beta \gamma}$.

4) $\omega_{\beta \gamma}$ is disjoint from any Weyl state and any plane wave.

Proof. 1) is obvious from theorem (2.18) and the maximality of the subgroup.

2) Is a consequence of Corollary (2.19).

3) Let $(x, p),\left(x^{\prime}, p^{\prime}\right) \in \mathbb{R}^{2}$; one has the following obvious calculation:

$$
\begin{aligned}
\left(\Omega_{\beta \gamma} \mid \pi_{\beta \gamma}\left(\delta_{x, p}\right) \pi_{\beta \gamma}\left(\delta_{x^{\prime}, p^{\prime}}\right) \pi_{\beta \gamma}\left(\delta_{-x,-p}\right) \Omega_{\beta \gamma}\right) & \\
= & \exp \left(-i\left(x p^{\prime}-p x^{\prime}\right)\right)\left(\Omega_{\beta \gamma} \mid \pi_{\beta \gamma}\left(\delta_{x^{\prime}, p^{\prime}}\right) \Omega_{\beta \gamma}\right) \\
= & 0 \text { if } \quad\left(x^{\prime}, p^{\prime}\right) \neq\left(n^{\prime} \lambda, \frac{2 \pi m^{\prime}}{\lambda}\right) \\
= & \exp \left(-i\left(x \frac{2 \pi m^{\prime}}{\lambda}-p n^{\prime} \lambda\right)+i \beta n^{\prime} \lambda+i \gamma \frac{2 \pi m^{\prime}}{\lambda}+i \pi m^{\prime} n^{\prime}\right) \\
& \text { if }\left(x^{\prime}, p^{\prime}\right)=\left(n^{\prime} \lambda, \frac{2 \pi m^{\prime}}{\lambda}\right) \\
= & \exp \left(i n^{\prime} \lambda(\beta+p)+i \frac{2 \pi m^{\prime}}{\lambda}(\gamma-x)+i \pi m^{\prime} n^{\prime}\right) .
\end{aligned}
$$

Hence the result.

Let us remark that there is an orthonormal basis of $\mathscr{H}_{\beta \gamma}$ which is indexed by the points in the rectangle $\Delta_{\lambda}$

$$
\Delta_{\lambda}=\left\{(\tilde{x}, \tilde{p}) ; \tilde{x} \in\left[0, \lambda\left[, \tilde{p} \in\left[0, \frac{2 \pi}{\lambda}\right]\right\} .\right.\right.
$$


Let us denote by $\mid \tilde{x}, \tilde{p})_{\beta \gamma},(\tilde{x}, \tilde{p}) \in \Delta_{\lambda}$, this basis; one has

$$
\mid \tilde{x}, \tilde{p})_{\beta \gamma}=\pi_{\beta \gamma}\left(\delta_{\tilde{x}, \tilde{p}}\right) \Omega_{\beta \gamma} .
$$

4) Since the $\pi_{\beta \gamma}$ 's are irreducible it is enough to prove the nonunitary equivalence of $\pi_{00}$ to the usual representation and to the presentation induced by the plane waves. This is achieved if one remarks that unitary equivalence conserves the continuity properties of

$$
\lambda \rightarrow \pi\left(\delta_{\lambda(x, p)}\right)
$$

for the usual representation

$$
x \rightarrow \pi\left(\delta_{x, p}\right)
$$

for the plane waves.

The $\mid \tilde{x}, \tilde{p})_{\beta \gamma}$ diagonalize the $\pi_{\beta \gamma}\left(\delta_{x, p}\right)$ for

in the following sense.

$$
(x, p)=\left(n \lambda, \frac{2 \pi m}{\lambda}\right), \quad(n, m) \in \mathbb{Z}^{2},
$$

Lemma (3.24). Within the representation $\pi_{\beta \gamma}$ one has

$$
\begin{aligned}
& \pi_{\beta \gamma}\left(\delta_{n \lambda, \frac{2 \pi m}{\lambda}}\right)(\tilde{x}, \tilde{p})_{\beta \gamma} \\
= & \left.\exp \left(-i\left(n \lambda \tilde{p}-\frac{2 \pi m}{\lambda} \tilde{x}\right)\right) \exp \left(i\left(n \lambda \beta+\frac{2 \pi m}{\lambda} \gamma+\pi m n\right)\right) \mid \tilde{x}, \tilde{p}\right)_{\beta \gamma} .
\end{aligned}
$$

Zak waves have the same relationship to the Zak $k \cdot q$ representation [11] that the plane waves have to the usual $p$ representation; that is the reason for which we choose the terminology. In what we have done we have considered special closed subgroups of $\left(\mathbb{R}^{2}, \xi\right)$, namely those whose generators are parallel to the axis or rectangular lattices parallel to the axis. As far as the existence and uniqueness, or the general features of invariant states with respect to closed abelian subgroups of $\left(\mathbb{R}^{2}, \xi\right)$ are concerned, this is sufficient to treat the general case as a result of the Lemma (3.3) and the trivial:

Lemma (3.25). Let $\omega$ be a state of $\overline{\Delta\left(\mathbb{R}^{2 N}, \xi\right)}$ (resp. pure state) invariant with respect to some closed $\xi$-abelian subgroup of $\left(\mathbb{R}^{2 N}, \xi\right) \Gamma$. Let $M$ be a symplectic transformation of $\left(\mathbb{R}^{2 N}, \xi\right)$ and $\tau_{M}$ the corresponding *-automorphism of $\overline{\Delta\left(\mathbb{R}^{2 N}, \xi\right)}$, then $\omega \circ \tau_{M}$ is an invariant state (resp. invariant pure state) with respect to the $\xi$-abelian subgroup of $\left(\mathbb{R}^{2 N}, \xi\right)$ $M^{-1} \Gamma=\left\{M^{-1} a ; a \in \Gamma\right\}$.

Because of the uniqueness theorem of Stone-von-Neumann [8] such a $*$-automorphism is automatically implemented within the usual Schrödinger representation: it does not change the continuity property 
of the state. This is no longer the case for the representation we have considered in this section as we shall see. Firstly let us give an obvious result.

Lemma (3.26). The group of symplectic transformations on $\left(\mathbb{R}^{2}, \xi\right)$ is isomorphic to the group of real two by two matrices of determinant one.

We can give now the following proposition.

Proposition (3.27). Let $\pi$ be the representation of $\overline{\Delta\left(\mathbb{R}^{2}, \xi\right)}$ induced by a pure plane wave (or a Bloch state); let $\tau_{M}$ be the *-automorphism of $\overline{\Delta\left(\mathbb{R}^{2}, \xi\right)}$ corresponding to a symplectic transformation $M$ of $\left(\mathbb{R}^{2}, \xi\right)$; the necessary and sufficient condition for $\tau_{M}$ to be unitarily implemented within $\pi$ is that $M$ leaves the axis $(x, 0)$ invariant or equivalently that $M$ is of the form

$$
M=\left(\begin{array}{ll}
\alpha & \beta \\
0 & \alpha^{-1}
\end{array}\right) \quad \begin{aligned}
& \alpha, \beta \in \mathbb{R} \\
& \alpha \neq 0
\end{aligned} .
$$

Proof. The sufficiency of the previous condition is clear. Indeed let $\omega$ be a pure Bloch state defined by the fact that it is invariant with respect to some subgroup $\Gamma_{a}=\{(n a, 0) ; n \in \mathbb{Z}\}$. It is pure and $x \rightarrow \omega\left(\delta_{x, p}\right)$ is continuous [cf. Theorem (3.13)]; thus from Lemma (3.25) $\omega \circ \tau_{M}$ is pure and $\omega \circ \tau_{M}$ is invariant with respect to

$$
M^{-1} \Gamma_{a}=\left\{M^{-1}(n a, 0)=\left(n \alpha^{-1} a, 0\right) ; n \in \mathbb{Z}\right\}=\Gamma_{\alpha^{-1} a} .
$$

Moreover $\omega \circ \tau_{M}\left(\delta_{x, p}\right)=\omega\left(\delta_{\alpha x+\beta p, \alpha^{-1} p}\right)$ and the function $x \rightarrow \omega \circ \tau_{M}\left(\delta_{x, p}\right)$ is still continuous; then, from Theorem (3.13) and Proposition (3.19), $\omega \circ \tau_{M}$ is a vector state of $\pi_{0}$ [cf. Proposition (3.6)]; hence (cf. [6], 2.4.6.) $\tau_{M}$ is unitarily implemented within $\pi_{0}$.

On the other hand assume that $M$ does not leave the axis $(x, 0)$ invariant

$$
M(x, 0)=(\alpha x, \gamma x)
$$

and moreover that it is unitarily implemented within $\pi_{0}$ so that there exists an unitary operator $U$ on $\mathscr{H}_{0}$ such that

$$
U \pi_{0}\left(\delta_{x, p}\right) U^{-1}=\pi_{0}\left(\tau_{M} \delta_{x, p}\right) .
$$

Let us restrict ourselves to $p=0$

$$
U \pi_{0}\left(\delta_{x, 0}\right) U^{-1}=\pi_{0}\left(\delta_{\alpha x, \gamma x}\right) .
$$

The left hand side is (weakly) continuous for every $x$ but the right hand side has a discontinuity at $x=0$ which is a contradiction.

One has already encountered this fact in Proposition (3.9) for the group of $x$-automorphisms associated to the group of symplectric 
transformations

$$
M_{t}=\left(\begin{array}{cc}
1 & t / m \\
0 & 1
\end{array}\right) \quad \begin{aligned}
& t \in \mathbb{R} \\
& m>0
\end{aligned} .
$$

Finally we are left with the study of relations between the Zak waves corresponding to different lattices. We shall not treat the general case but only the case of a rectangular lattice. The next result shows how the equivalence classes of the representations depend in a non-smooth manner on the shape of the lattice, which is a serious drawback for the physical interpretation of the theory.

Proposition (3.29). Let $\omega_{1}$ and $\omega_{2}$ be two Zak waves corresponding to two different rectangular lattices along the axis of basis $a_{i}$ respectively; let $\chi_{i}$ the corresponding characters, let $\pi_{i}, \mathscr{H}_{i}, \Omega_{i}$ the corresponding G.N.S. triplets; then $\pi_{1}$ is unitarily equivalent to $\pi_{2}$ if and only if the ratio $a_{1} / a_{2}$ is rational.

Proof. According to Proposition (3.23) we can restrict ourselves to the case where $\chi_{i}\left(n a_{i}, \frac{2 \pi m}{a_{i}}\right)=(-1)^{m n}$ for $m, n \in \mathbb{Z}$. Then the nesessary and sufficient condition for the equivalence of $\pi_{1}$ and $\pi_{2}$ is the existence of a vector $\Phi \in \mathscr{H}_{1}$ such that

$$
\pi_{1}\left(\delta_{n a_{2}, \frac{2 \pi m}{a_{2}}}\right) \Phi=(-1)^{n m} \Phi \quad n, m \in \mathbb{Z}
$$

(see $[6], 2.4 .6$.) since by Theorem $(2.18)$ the vector state $\omega_{\Phi}$ is equal to $\omega_{2}$.

As we noticed previously, $\Phi$ can be written

$$
\Phi=\sum_{m, n} f(n, m) \pi_{1}\left(\delta_{\tilde{x}_{n}, \tilde{p}_{m}}\right) \Omega_{1}
$$

with $\sum_{m, n}|f(n, m)|^{2}<\infty$ and $\left(\tilde{x}_{n}, \tilde{p}_{m}\right) \in \Delta_{a_{1}}$ and the $\pi_{1}\left(\delta_{\tilde{x}_{n}, \tilde{p}_{n}}\right) \Omega_{1}$ linearly independent.

Up to a trivial phase factor, the $\pi_{1}\left(\delta_{n a_{2}}, \frac{2 \pi m}{a_{2}}\right)$ permute the set of $\pi_{1}\left(\delta_{\tilde{x}_{n}, \tilde{p}_{m}}\right) \Omega_{1}$, hence in view of the invariance of $\Phi$ and the normalizability of $\Phi$ the sum (3.31) has to be finite and given $\tilde{x}_{i}$ there exist $p$ and $q$ integers such that

hence the result

$$
\tilde{x}_{i}+p a_{2}=\tilde{x}_{i}+q a_{1}
$$

$$
a_{1} / a_{2}=p / q \text {. }
$$

Consequently we have proved the necessity of (3.32). In order to prove the sufficiency, let us consider the case where $q=1$ since the 
general case can be dealt by a repeated application of this reasoning; the vector $\Phi$ which satisfies (3.30) is easy to construct

$$
\Phi=\frac{1}{\sqrt{p}} \sum_{j=1}^{p-1} \pi_{1}\left(\delta_{\frac{j}{p} a_{1}, 0}\right) \Omega_{1} .
$$

Acknowledgments. It is a pleasure to us to thank Drs. A. Grossmann, D. Testard, and A. Verbeure for illuminating discussions, and Prof. H. Araki for pointing us some errors in an earlier version of this paper and suggesting improvements.

\title{
References
}

1. Sirugue, M.: Improper states of canonical communication relation for a finite number of degrees of freedom. Preprint, Marseille (1971)

2. Manuceau, J.: Ann. Inst. Henri Poincaré VIII (2), 139 (1968)

3. Manuceau,J., Sirugue,M., Testard,D., Verbeure,A.: Commun. math. Phys. 32, $231-243$ (1973)

4. Slawny, J.: On factor representation and the $C^{*}$-algebra of C.C.R. Preprint, Göttingen (1971)

5. Naimark, M.A.: Normed rings. Groningen: Wolters-Noordhoff Publishing 1970

6. Dixmier, J.: Les $C^{*}$-algèbres et leurs représentations. Paris: Gauthier-Villars 1969

7. Kittel, C.: Introduction to solid state physics. New York: John Wiley-Sons 1959

8. von Naumann, J.: Math. Ann. 104, 570 (1931)

9. Mackey, G. W.: Duke Math. J. 16, 313-326 (1949)

10. Slawny, J.: On the regular representation, von Neumann uniqueness theorem and the $C^{*}$-algebra of canonical commutation and anticommutation relations. Preprint, Tel Aviv (1971)

11. Zak, J.: Phys. Rev. 168, 686 (1968)

Communicated by H. Araki

\author{
R. Beaume \\ J. Manuceau \\ A. Pellet \\ M. Sirugue \\ Centre de Physique Théorique \\ C.N.R.S \\ 31, chemin J. Aiguier \\ F-13274 Marseille Cedex 2, France
}


